\title{
On Locally Inner Automorphisms of Certain $C^{*}$-algebras
}

\author{
Sin-ei TAKAHASI*
}

The purpose of this note is to extend, in case $T$ is a locally compact Hausdorff space, the results of Smith [1] concerning locally inner automorphisms of $C(T, L(H)), \mathrm{C}^{*}$-algebra consisting of continuous $L(H)$ valued functions on a compact Hausdorff space $T$.

1. An automorphism of a $C^{*}$-algebra $B$ means a $*$-isomorphism of $B$. The set of all automorphisms of $B$ is denoted by Aut $(B)$. Aut $(B)$ is a group in a natural way, the group operation being a composition of mappings. Moreover, Aut $(B)$ is a topological group with uniform topology, that is

$$
\|\alpha\|=\sup \{\|\alpha(x)\|: x \in B,\|x\| \leq 1\} .
$$

Let $\mathrm{CF}(B)$ be the group of all center-fixing automorphisms of $B$ (i.e. $\alpha \in$ Aut $(B), \alpha(x)=x$ for all $x \in Z(B)$ (the center of $B$ )). Let $T$ be a locally compact Hausdorff space, $\mathfrak{B}=\left(\left(B(t)_{t \in T}, \Theta\right)\right.$ the continuous field of $C^{*}$ algebras on $T$. Let $B$ be the $C^{*}$-algebra defined by $\mathfrak{B}$. If $t \in T$ we denote by

$$
e_{\iota}: B \rightarrow B(t)
$$

the mapping given by $e_{t}(x)=x(t)$.

Lemma 1. 1. If $\alpha \in \operatorname{CF}(B)$ and $t \in T$, then $\alpha\left(\operatorname{Ker} e_{t}\right) \subset \operatorname{Ker} e_{t}$.

Proof. Let $M_{\imath}$ be the maximal ideal of $C_{0}(T)$, the algebra of all continuous functions on $T$ which vanish at infinity, determined by $t \in T, i$. e. $M_{t}=\left\{f \in C_{0}(T): f(t)=0\right\}$. We assert that

Ker $e_{\iota}=$ the uniform closure of $\left\{f \cdot x: f \in M_{\iota}, x \in B\right\}$. If $x \in \operatorname{Ker} e_{\iota}$,

Received October 26, 1972.

*Department of Mathematics, Faculty of Science, Ibaraki University, Mito. 
then for any $\varepsilon>0$, there is a compact neighbourhood $U_{t}$ of $t$ such that $\|x(s)\| \leq \varepsilon / 2$ for any $s \in U_{t}$. Let $K$ be a compact subset of $T$ such that $K \supset U_{t}$ and $\|x(s)\| \leq \varepsilon / 2$ for any $s \in T \backslash K$. Let $f \in M_{t}$ be a function on $T$ such that $0 \leq f\left(K \backslash U_{t}\right)=1$. Then we have

$$
\begin{aligned}
\|f \cdot x-x\|= & \sup \{\|f(s) x(s)-x(s)\|: s \in T \backslash K\} \\
& +\sup \left\{|f(s)-1|\|x(s)\|: s \in U_{\imath}\right\} \leq \varepsilon,
\end{aligned}
$$

and hence $x \in \overline{M_{t} \cdot B}$ where $M_{t} \cdot B=\left\{f \cdot x: f \in M_{t}, x \in B\right\}$.

Conversely, a limit point of $M_{t} \cdot B$ is the element of $\operatorname{Ker} e_{t}$, since the evaluation mapping $e_{t}: B \rightarrow B(t)$ is continuous.

If $f \in M_{t}$ and $x \in B$, then

$$
\alpha(f \cdot x)=\alpha((f \cdot 1) \cdot x)=\alpha(f \cdot 1) \alpha(x)=f \cdot \alpha(x) .
$$

Thus $\alpha(f \cdot x) \in \operatorname{Ker} e_{t}$. Therefore we have $\alpha\left(\operatorname{Ker} e_{t}\right) \subset \operatorname{Ker} e_{t}$ since $\alpha$ is uniformly continuous.

Let $\alpha \in \mathrm{CF}(B), \quad a \in B(t)$ and $t \in T$. Then there is $x \in B$ with $x(t)=a$ and we define a function $\tilde{\alpha}_{t}: B(t) \rightarrow B(t)$ by

$$
\tilde{\alpha}_{\imath}(a)=e_{t}(\alpha(x))
$$

This is well defined by Lemma 1.1 .

Lemma 1. 2. If $\alpha, \beta \in \operatorname{CF}(B)$ and $t \in T$, then $(\alpha \beta)_{t}^{-}=\tilde{\beta}_{t} \tilde{\beta}_{t}$.

Proof. For any $x \in B$, we have

$$
\begin{aligned}
(\alpha \beta)_{t}(e(x)) & =e_{t}\left(\alpha(\beta(x))=\tilde{\alpha}_{t}\left(e_{t}(\beta(x))\right)\right. \\
& =\tilde{\alpha_{t}} \tilde{\beta}_{t}\left(e_{t}(x)\right) .
\end{aligned}
$$

Proposition 1. 3. If $\alpha \in \mathrm{CF}(B)$ and $t \in T$, then $\tilde{\alpha}_{t} \in \operatorname{Aut}(B(t))$.

Proof. We can easily show that $\tilde{\alpha}_{t}$ is the *homomorphism of $B(t)$. By Lemma 1. 2 it follows that $\left(\tilde{\alpha}^{-1}\right)_{t}=\left(\tilde{\alpha}_{t}\right)^{-1}$. Then $\tilde{\alpha}_{t}$ is the automorphism of $B(t)$.

Let $\Pi_{t \in T} \operatorname{Aut}(B(t))$ be a product of topological groups of automorphisms of $C^{*}$-algebras. It is the group under the pointwise multi- 
plication. $\Pi_{t \in T}$ Aut $(B(t))$ becomes a topological group with the topology determind by the system of neibourhoods of points $\varphi$ in $\Pi_{t \in T}$ Aut $(B(t))$ having the form,

$$
U(\varphi, \varepsilon)=\left\{\varphi^{\prime} \in \Pi_{t \in T} \text { Aut }(B(t)): \sup _{t \in T}\left\|\varphi_{t}-\varphi_{t}^{\prime}\right\|<\varepsilon\right\}
$$

where $\varepsilon>0$. We shall regard $\tilde{\alpha}$ as an element of $\Pi_{t \in T}$ Aut $(B(t))$.

Proposition 1. 4. A map $\alpha \rightarrow \tilde{\alpha}$ is the isomorphism of the topological group CF (B) into the topological group $\Pi_{t \in T} \operatorname{Aut}(B(t))$ and the image $\mathrm{CF}(B)$ is closed.

Proof. By Lemma 1. 2 it follows that $\alpha \rightarrow \tilde{\alpha}$ is the homomorphism of the group $\mathrm{CF}(B)$ into the group $\Pi_{t \in T}$ Aut $(B(t))$. Let $\alpha, \beta \in \mathrm{CF}(B)$. We have

$$
\begin{aligned}
\sup _{t \in T}\left\|\tilde{\alpha}_{t}-\tilde{\beta}_{t}\right\| & \geq \sup _{t \in T} \sup _{t x 1 \leq 1}\left\|e_{t}(\alpha-\beta)(x)\right\| \\
& =\|\alpha-\beta\| .
\end{aligned}
$$

Then $\alpha \rightarrow \tilde{\alpha}$ is open and injective.

Let $x \in B$ with $\|x(t)\| \leq 1$ where $t \in T$. Then there is a compact neighbourhoods $U_{t}$ of $t$ such that $\left\|x_{s}\right\| \leq 2$ on $U_{t}$. Let $f \in C_{0}(T)$ such that $0 \leq f \leq 1, f\left(T \backslash U_{t}\right)=0$ and $f(t)=1$, so that $e_{t}(f \cdot x)=x(t)$ and i| $f \cdot x \| \leq 2$. Then we have

$$
\begin{aligned}
\sup _{t \in T}\left\|\tilde{\alpha}_{t}-\tilde{\beta}_{t}\right\| & \leq \sup _{t \in T} \sup _{\mid x \otimes \leq 2}\left\|e_{t}(\alpha-\beta)(x)\right\| \\
& \leq 2\|\alpha-\beta\|
\end{aligned}
$$

Thus $\alpha \rightarrow \tilde{\alpha}$ is continuous.

Finally, suppose that a net $\left\{\tilde{\alpha}^{{ }}{ }_{l}\right\} \subset \mathrm{CF}(B)^{-}$convergents to $\varphi \in$ $\Pi_{t \in T}$ Aut $(B(t))$. Then, for any $\varepsilon>0$, there exists $\lambda_{0}$ such that $\sup _{t \in T}\left\|\tilde{\alpha}_{t}-\varphi_{t}\right\|<\varepsilon$ for every $\lambda \geq \lambda_{0}$ We define $\alpha(x)(t)=\varphi_{t}(x(t))$ for any $t \in T, x \in T$. A simple computation implies that $\alpha \in \mathrm{CF}(B)$ and $\tilde{\alpha}=\varphi$ and so $\operatorname{CF}(B)$ is closed.

2. Let $S$ be a compact subset of the locally compact Hausdorff space $T$ and $\mathfrak{B}$ is a continuous field induced by $\mathfrak{B}$ on $S$ where $\mathfrak{B}=$ 
$\left(\left(B(t)_{t \in \mathrm{T}}, \theta\right)\right.$ is a continuous field of $\mathrm{C}^{*}$-algebras on $T$ [2]. Let $B \mid s$ be the $\mathrm{C}^{*}$-aigebra defined by $\mathfrak{B}_{\mid s}$ where $B$ is the $\mathrm{C}^{*}$-algebra defined by $B$. By Corollaire 11. 5 in [2], the following restriction map $\rho$ is the *-homomorphism of $B$ onto $B \mid s$,

$$
\left.\rho(X)=x \text { where } X \in B \text { and } x=X_{\mid s}=(X(t))_{t \in S}\right) .
$$

Definition. If $\alpha \in \mathrm{CF}(B)$ and $S$ is a compact subset of $T$, then we define $\alpha_{\mid s}: B|s \rightarrow B| s$ by $\alpha|s(x)=\alpha(X)| s$ where $X \in B$ is any extension of $X$. This is well defined by Lemma 1. 1 .

The following diagram is commutative where $\alpha \in \operatorname{CF}(B)$ :

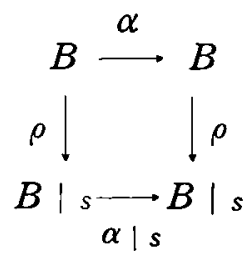

Definition. If $\alpha \in \operatorname{CF}(B)$, then $\alpha$ is called locally inner iff there exists a relatively compact open covering $\left(T_{i}\right)_{i \in I}$ on $T$ such that $\alpha \mid \bar{T}_{i}$ is inner for all $i \in I$ where $\bar{T}_{i}$ is the closure of $T_{i}$. The set of all locally inner automorphisms is denote by $\operatorname{loc} \cdot \operatorname{Inn}(B)$.

We note that if $\mathfrak{B}$ is a constant field on a compact Hausdorff space $T$, then the above definition for locally inner automorphisms is nothing but the definition of Smith [1] (p 631).

Proposition 2. 1. The loc-Inn(B) is a subgroup of $\operatorname{Aut}(B)$.

Proof. We obtain easily the result above, applying the proof of Propositition 3. 4 in [1]

3. Let $T$ be a locally compact Hausdorff space and $H$ a Hibert space. Let $L(H)$ be the algebra of all continuous linear operators on $H$. Let $B=C_{0}(T, L(H))$. the algebra of all continuous functions $x: T$ $\rightarrow L(H)$ which vanish at infinity. Then $B$ is the $\mathrm{C}^{*}$-algebra defined by the constant field $\mathfrak{B}$ on $T$ defined by $L(H)$.

Lemma 3. 1. Let $T, B$, and $H$ be as above. Let $S$ be a compact subset of $T$. Then if $\alpha \in \operatorname{CF}(B)$, then $\alpha \mid s \in \operatorname{CF}(B \mid s)$ and $(\alpha \mid s)^{\sim}=$ $\tilde{\alpha} \mid s$. 
Proof. Let $a$ be a center element of $B \mid s$. Note that $B \mid s$ $=C(S, L(H))$, the algebra of all continuous functions $a: S \rightarrow L(H)$. Then there exists $f \in C(S)$ such that $a=f \cdot 1$ where 1 is the identity of $L(H)$. Let $f_{0} \in C_{0}(T)$ with $f=f_{0} \mid s$, so that $f_{0} \cdot 1$ is the center element of $B$. Hence,

$$
\alpha\left|s(a)=\alpha\left(f_{0} \cdot 1\right)\right| s=\left(f_{0} \cdot 1\right) \mid s=a .
$$

By the diagram of the section 2, we see that $(\alpha \mid s)^{-}=\tilde{\alpha} \mid s$.

THEOREM 3. 2. If $\alpha \in \mathrm{CF}(B)$ then $\tilde{\alpha}: T \rightarrow$ Aut $(L(H))$ is continuous in the uniform operator topology iff $\alpha \in \operatorname{loc} \cdot \operatorname{Inn}(B)$.

Proof. Suppose that $\alpha \in \operatorname{loc} \operatorname{Inn}(B)$ and let $\left(T_{i}\right)_{t \in I}$ be a relatively compact open covers of $\mathrm{T}$ such that $\alpha_{\mid} \bar{T}_{i}$ is inner for all $i \in I$. By the proposition 3. 16 in [1] and Lemma 3. $1, \tilde{\alpha}_{i} \bar{T}_{i}: \bar{T}_{\mathfrak{i}} \rightarrow \operatorname{Aut}(L(H))$ is continuous. Therefore $\tilde{\alpha}$ is continuous.

Conversely, suppose that $\tilde{\alpha}: T \rightarrow \operatorname{Aut}(L(H))$ is continuous. Let $\left(T_{i}\right)_{t \in I}$ be a relatively compact open covers of $T$. By Lemma 3. 1 $\left(\alpha \mid \bar{T}_{i}\right)^{-}$is continuous map of $\bar{T}_{i}$ into $\operatorname{Aut}(L(H))$. Then we have $\alpha_{\mid} \bar{T}_{i} \in \operatorname{loc} \cdot \operatorname{Inn}\left(B \mid \bar{T}_{i}\right)$ for all $i \in I$ by the theorem 3. 12 in [1]. Therefore $\alpha \in$ loc-Inn $(B)$ by the note in the section 2 .

We can see that $C(T$, Aut $(L(H))$, the group of all continuous functions of $\mathrm{T}$ into $\operatorname{Aut}(L(H))$, is the topological subgroup of $\Pi_{t \in T} \operatorname{Aut}(L(H))$.

THEOREM 3. 3. $\sim: \operatorname{loc}-\operatorname{Inn}(B) \rightarrow C(T, \operatorname{Aut}(L(H))$ is an isomorphism of topological groups.

Proof. We shall use the technique in the proof of the theorem 3. 5 in [1]. Let $\varphi \in C(T$, Aut $(L(H)))$. For any $x \in B$ we define $\hat{\varphi}(x)(t)=\varphi(t)(x(t))$ for any $t \in T$.

Note that

$\|\varphi(t)(x(t))-\varphi(s)(x(s))\| \leq\|x(t)-x(s)\|+\|\varphi(t)-\varphi(s)\|\|x(s)\|$

for $x \in B$, and $t, s \in T$. Then $\hat{\varphi}(x)$ is a continuous map of $T$ into $L(H)$ and we can easily show that $\hat{\varphi}(x)$ vanishes at infinity. Hence, 
$\hat{\varphi}$ defines an automorphism of $B$. Note that the center of $B=C_{0}(T) \cdot 1$. Then $\hat{\varphi} \in \operatorname{CF}(B)$ by the definition of $\wedge$. For any $x \in B$ and $t \in T$ we have

$$
\hat{\varphi}^{\sim}(t)(x(t))=\hat{\varphi}(x)(t)=\varphi(t)(x(t)) .
$$

Then $\hat{\varphi}^{\sim}=\varphi$, so that $\hat{\varphi} \in$ loc-Inn $(B)$ by Theorem 3. 2.

On the other hand, let $\alpha \in$ loc-Inn (B). For any $x \in B$ and $t \in T$, $(\tilde{\alpha})^{\hat{n}}(x)(t)=(\tilde{\alpha})(t)(x(t))=\alpha(x)(t)$. Then $(\tilde{\alpha})=\alpha$. Therefore $\sim$ and $\wedge$ are inverse isomorphism of abstract groups and the result follows from Proposition 1. 4.

COROLlaRY 3. 4. If $T$ is a separable locally compact Hausdorff space, then $\mathrm{CF}(B)=\operatorname{loc}-\operatorname{Inn}(B)$.

Proof. Let $\alpha \in \mathrm{CF}(B)$ and $a \in L(H)$. Let $t \in T$ and $U_{\iota}$ a compact neighbourhood of $t$. There is $x \in B$ such that $x(s)=a$ for all $s \in U_{t}$. Then we have

$$
\left\|\tilde{\alpha}_{t}(a)-\tilde{\alpha}_{s}(a)\right\|=\|\alpha(x)(t)-\alpha(x)(s)\|
$$

for all $s \in U_{\iota}$. Then $\tilde{\alpha}: T \rightarrow \operatorname{Aut}(L(H))$ is strongly continuous. Applying Kallmann's theorem (Theorem F in [1]) we can show our corollary (cf. Proof of Theorem 3. 11 in [1]).

\section{References}

[1] M. B. Smith, On automorphism groups of $C^{*}$-algebras, Trans. Amer, Math. Soc. 152 (1970), 623-648.

[2] J. Dixmier, Les $C^{*}$-algèbre et leur représentations, Gauthier-Villars, Paris, 1964. 